\title{
Tandem Oxidation/Halogenation of Aryl Allylic Alcohols Under Moffatt- Swern Conditions
}

\author{
Jiandong Yin, Christina E. Gallis, and John D. Chisholm* \\ Department of Chemistry, 1-014 Center for Science and Technology \\ Syracuse University, Syracuse, NY 13244 \\ SUPPORTING INFORMATION
}

CONTENTS:

$\begin{array}{ll}\text { General Experimental Details } & \text { S2 }\end{array}$

$\begin{array}{ll}\text { Representative Procedure } & \text { S3 }\end{array}$

$\begin{array}{ll}\text { Tabulated Characterization Data } & \text { S3-S8 }\end{array}$

$\begin{array}{ll}\text { References } & \text { S9 }\end{array}$

$\begin{array}{ll}\text { NMR Spectra } & \text { S10-S28 }\end{array}$ 
General. NMR spectra were recorded on solutions in deuterated chloroform $\left(\mathrm{CDCl}_{3}\right)$, with residual tetramethylsilane ( $\delta 0.00 \mathrm{ppm}$ for $\left.{ }^{1} \mathrm{H} \mathrm{NMR}\right)$ and residual chloroform $\left(\delta 77.23 \mathrm{ppm}\right.$ for $\left.{ }^{13} \mathrm{C} \mathrm{NMR}\right)$ as the internal reference, and were reported in parts per million $(\mathrm{ppm})$. Infrared spectra were obtained from samples prepared as thin films between $\mathrm{NaCl}$ plates. High resolution mass spectra were obtained using the positive ion electrospray ionization mode with $\mathrm{MeOH} / \mathrm{THF}+\mathrm{NaCl}$ as solvent. Flash column chromatography was carried out with $(40-63 \mu \mathrm{m})$ flash silica gel. Analytical thin layer chromatography (TLC) was performed on precoated glass backed plates (silica gel $60 \mathrm{~F}_{254} ; 0.25 \mathrm{~mm}$ thickness). All anhydrous reactions were run under a positive pressure of argon or nitrogen. All syringes, and hypodermic needles, and reaction flasks required for anhydrous reactions were dried in an oven and cooled under a $\mathrm{N}_{2}$ atmosphere or in a desiccator. Dichloromethane and THF were dried by passage through an alumina column following the method of Grubbs. ${ }^{1}$ Triethylamine and DMSO were distilled from $\mathrm{CaH}_{2}$ and stored over $4 \AA$ molecular sieves. Oxalyl chloride was refluxed and distilled from quinoline. All other reagents and solvents were purchased from commercial sources and used without further purification.

The aryl allylic alcohols used as starting materials were prepared via the reaction of vinylmagnesium chloride and the corresponding aldehydes. Allylic alcohols $\mathbf{1}$ and $\mathbf{4 1}$ were purchased from commercial sources. Characterization data for allylic alcohols $7,{ }^{2} \mathbf{1 0},{ }^{3,4} \mathbf{1 3},{ }^{5} \mathbf{1 6},{ }^{5} \mathbf{1 9},{ }^{6} \mathbf{2 2},{ }^{7} \mathbf{2 5},{ }^{8}$ $\mathbf{2 8},{ }^{9} \mathbf{3 1},{ }^{10} \mathbf{3 4},{ }^{8} \mathbf{3 9}^{11}$ and $\mathbf{4 3}^{12}$ has been previously reported in the literature. Characterization data for enones $2,^{13} \mathbf{4 0}{ }^{14}$ and $\mathbf{4 2}{ }^{15}$, allylic chlorides $32^{16}$ and $\mathbf{4 4}{ }^{17}$ as well as 1-(4-tolyl)-2-propen-1-ol ${ }^{18}$ and 1 [(1E)-3-chloro-1-propenyl]-4-methyl-benzene ${ }^{19}$ have also been previously reported. Purity of known compounds was established using ${ }^{1} \mathrm{H}$ NMR. 


\section{General Procedures for the formation of $\alpha$-Halo Ketones}

A flame-dried flask was charged with dry $\mathrm{CH}_{2} \mathrm{Cl}_{2}$ and cooled to $-78^{\circ} \mathrm{C}$ with a dry ice/acetone bath.

Oxalyl chloride or oxalyl bromide (3 equiv) was then added followed by DMSO (4 equiv). The reaction was kept at $-78^{\circ} \mathrm{C}$ for $15 \mathrm{~min}$, after which time the allylic alcohol (1 equiv dissolved in $\mathrm{CH}_{2} \mathrm{Cl}_{2}$ ) was added. After addition of the allylic alcohol the concentration of the reaction was approximately $0.1 \mathrm{M}$ in regards to the allylic alcohol. The reaction was kept at $-78^{\circ} \mathrm{C}$ for $1 \mathrm{~h}$, after which time triethylamine (6 equiv) was added and the reaction allowed to warm to room temperature over $30 \mathrm{~min}$. The reaction mixture was then poured over ice-cold $1 \mathrm{M} \mathrm{HCl}$ and extracted with hexanes. The combined extracts were dried $\left(\mathrm{Na}_{2} \mathrm{SO}_{4}\right)$ and concentrated. Purification of the residue by silica gel chromatography provided the halogenated vinyl ketones or allylic chloride.

The same general procedure was used to generate the following $\alpha$-halo ketones and allylic chlorides.

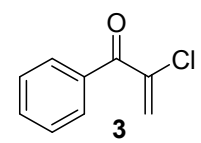

TLC $R_{f}=0.43\left(10 \%\right.$ ethyl acetate/hexanes); IR (film from $\left.\mathrm{CH}_{2} \mathrm{Cl}_{2}\right)$ 3064, 3030, 2928, 1674, 1601, $1385 \mathrm{~cm}^{-1} ;{ }^{1} \mathrm{H}$ NMR $\left(300 \mathrm{MHz}, \mathrm{CDCl}_{3}\right) \delta$ 7.77-7.83 (m, 2H), 7.56-7.64 (m, 1H), 7.44-7.52 (m, $\left.2 \mathrm{H}\right)$, $6.29(\mathrm{~d}, J=1.8 \mathrm{~Hz}, 1 \mathrm{H}), 6.09(\mathrm{~d}, J=1.8 \mathrm{~Hz}, 1 \mathrm{H}) ;{ }^{13} \mathrm{C} \mathrm{NMR}\left(75 \mathrm{MHz}, \mathrm{CDCl}_{3}\right) \delta 190.0,138.7,135.8$, $133.3,129.8,128.7,126.6$. This compound has been previously reported. ${ }^{20}$

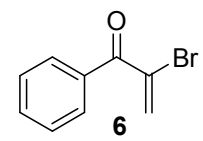

TLC $R_{f}=0.42\left(10 \%\right.$ ethyl acetate/hexanes); IR (film from $\left.\mathrm{CH}_{2} \mathrm{Cl}_{2}\right) 3065,1672,1597 \mathrm{~cm}^{-1} ;{ }^{1} \mathrm{H}$ NMR $\left(300 \mathrm{MHz}, \mathrm{CDCl}_{3}\right) \delta$ 7.78-7.84 (m, 2H), 7.45-7.60 (m, 1H), 7.44-7.50 (m, 2H), $6.54(\mathrm{~d}, J=2.4 \mathrm{~Hz}$ 
1H), $6.47(\mathrm{~d}, J=2.4 \mathrm{~Hz}, 1 \mathrm{H}) ;{ }^{13} \mathrm{C} \mathrm{NMR}\left(75 \mathrm{MHz}, \mathrm{CDCl}_{3}\right) \delta 190.4,135.3,133.4,130.4,129.9,129.8$, 128.7. Anal calcd for $\mathrm{C}_{9} \mathrm{H}_{7} \mathrm{BrO}$ : C, 51.22; H, 3.34. Found: $\mathrm{C}, 51.30 ; \mathrm{H}, 3.23$.

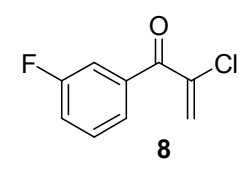

TLC $R_{f}=0.40$ (10\% ethyl acetate/hexanes); IR (film from $\left.\mathrm{CH}_{2} \mathrm{Cl}_{2}\right) 3074,1678,1587,1483,1440 \mathrm{~cm}^{-1}$; ${ }^{1} \mathrm{H}$ NMR $\left(300 \mathrm{MHz}, \mathrm{CDCl}_{3}\right) \delta 7.58(\mathrm{dt}, J=7.8,1.5 \mathrm{~Hz}, 1 \mathrm{H}), 7.43-7.51(\mathrm{~m}, 2 \mathrm{H}), 7.27-7.30(\mathrm{~m}, 1 \mathrm{H})$, $6.31(\mathrm{~d}, J=2.1 \mathrm{~Hz}, 1 \mathrm{H}), 6.12(\mathrm{~d}, J=2.1 \mathrm{~Hz}, 1 \mathrm{H}) ;{ }^{13} \mathrm{C} \mathrm{NMR}\left(75 \mathrm{MHz}, \mathrm{CDCl}_{3}\right) \delta 188.6(\mathrm{~d}, J=2.2 \mathrm{~Hz})$, $162.6(\mathrm{~d}, J=247 \mathrm{~Hz}), 138.3,137.7(\mathrm{~d}, J=6.7 \mathrm{~Hz}), 130.4(\mathrm{~d}, J=7.8 \mathrm{~Hz}), 126.9,125.5(\mathrm{~d}, J=3.1 \mathrm{~Hz})$, $120.3(\mathrm{~d}, J=21.3 \mathrm{~Hz}), 116.5(\mathrm{~d}, J=22.8 \mathrm{~Hz})$. Anal calcd for $\mathrm{C}_{9} \mathrm{H}_{6} \mathrm{ClFO}: \mathrm{C}, 58.56$; H, 3.28. Found: $\mathrm{C}$, $58.32 ; \mathrm{H}, 3.38$.

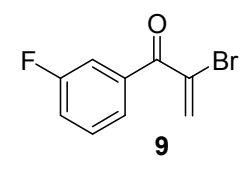

TLC $R_{f}=0.46$ (10\% ethyl acetate/hexanes); IR (film from $\left.\mathrm{CH}_{2} \mathrm{Cl}_{2}\right) 3061,1674,1586,1482,1438 \mathrm{~cm}^{-1}$; ${ }^{1} \mathrm{H}$ NMR $\left(300 \mathrm{MHz}, \mathrm{CDCl}_{3}\right) \delta$ 7.57-7.63 (m, 1H), 7.41-7.54 (m, 2H), 7.25-7.35 (m, 1H), $6.56(\mathrm{~d}, J=$ $2.4 \mathrm{~Hz}, 1 \mathrm{H}), 6.49(\mathrm{~d}, J=2.4 \mathrm{~Hz}, 1 \mathrm{H}) ;{ }^{13} \mathrm{C} \mathrm{NMR}\left(75 \mathrm{MHz}, \mathrm{CDCl}_{3}\right) \delta 189.0,162.7(\mathrm{~d}, J=248 \mathrm{~Hz})$, $137.3(\mathrm{~d}, J=6.6 \mathrm{~Hz}), 130.9,130.5(\mathrm{~d}, J=7.7 \mathrm{~Hz}), 129.2,125.6(\mathrm{~d}, J=3.0 \mathrm{~Hz}), 120.4(\mathrm{~d}, J=21.4 \mathrm{~Hz})$, $116.6(\mathrm{~d}, J=22.8 \mathrm{~Hz})$. Anal calcd for $\mathrm{C}_{9} \mathrm{H}_{6} \mathrm{BrFO}: \mathrm{C}, 47.19 ; \mathrm{H}, 2.64$. Found: $\mathrm{C}, 47.36 ; \mathrm{H}, 2.81$.

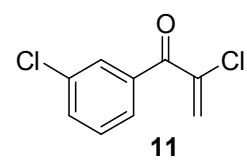

TLC $R_{f}=0.40$ (10\% ethyl acetate/hexanes); IR (film from $\left.\mathrm{CH}_{2} \mathrm{Cl}_{2}\right) 3069,1680,1598,1570,1421 \mathrm{~cm}^{-1}$; ${ }^{1} \mathrm{H}$ NMR $\left(300 \mathrm{MHz}, \mathrm{CDCl}_{3}\right) \delta 7.76(\mathrm{t}, J=1.8 \mathrm{~Hz}, 1 \mathrm{H}), 7.66(\mathrm{dt}, J=7.8,1.2 \mathrm{~Hz}, 1 \mathrm{H}), 7.56$ (ddd, $J=$ 
8.1, 2.1, 1.2 Hz, 1H), $7.42(\mathrm{t}, J=7.8 \mathrm{~Hz}, 1 \mathrm{H}), 6.31(\mathrm{~d}, J=2.1 \mathrm{~Hz}, 1 \mathrm{H}), 6.11(\mathrm{~d}, J=2.1 \mathrm{~Hz}, 1 \mathrm{H}) ;{ }^{13} \mathrm{C}$ $\operatorname{NMR}\left(75 \mathrm{MHz}, \mathrm{CDCl}_{3}\right) \delta 188.6,138.3,137.4,135.0,133.2,130.0,129.6,127.8,127.1$. Anal calcd for $\mathrm{C}_{9} \mathrm{H}_{6} \mathrm{Cl}_{2} \mathrm{O}: \mathrm{C}, 53.77 ; \mathrm{H}, 3.01$. Found: C, 53.60; H, 3.39.

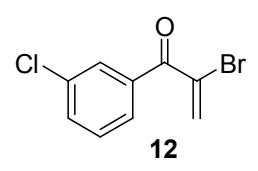

TLC $R_{f}=0.47\left(10 \%\right.$ ethyl acetate/hexanes); IR (film from $\left.\mathrm{CH}_{2} \mathrm{Cl}_{2}\right) 3066,1674,1595,1568,1469 \mathrm{~cm}^{-1}$; ${ }^{1} \mathrm{H}$ NMR $\left(300 \mathrm{MHz}, \mathrm{CDCl}_{3}\right) \delta 7.77(\mathrm{~m}, 1 \mathrm{H}), 7.67(\mathrm{~m}, 1 \mathrm{H}), 7.56(\mathrm{~m}, 1 \mathrm{H}), 7.42(\mathrm{t}, J=7.5 \mathrm{~Hz}, 1 \mathrm{H}), 6.57$ $(\mathrm{d}, J=2.4 \mathrm{~Hz}, 1 \mathrm{H}), 6.49(\mathrm{~d}, J=2.4 \mathrm{~Hz}, 1 \mathrm{H}) ;{ }^{13} \mathrm{C} \mathrm{NMR}\left(75 \mathrm{MHz}, \mathrm{CDCl}_{3}\right) \delta 189.0,136.9,135.0,133.3$, 131.0, 130.0, 129.8, 129.2, 128.0. Anal calcd for $\mathrm{C}_{9} \mathrm{H}_{6} \mathrm{BrClO}$ : C, 44.03; $\mathrm{H}, 2.46$. Found: C, 44.29; $\mathrm{H}$, 2.63.

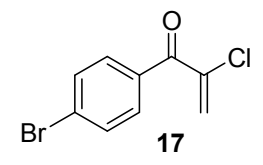

TLC $R_{f}=0.54\left(10 \%\right.$ ethyl acetate/hexanes); IR (film from $\left.\mathrm{CH}_{2} \mathrm{Cl}_{2}\right) 3079,1663,1584,1398 \mathrm{~cm}^{-1} ;{ }^{1} \mathrm{H}$ NMR $\left(300 \mathrm{MHz}, \mathrm{CDCl}_{3}\right) \delta 7.68(\mathrm{~d}, J=9.0 \mathrm{~Hz}, 2 \mathrm{H}), 7.61(\mathrm{~d}, J=9.2,2 \mathrm{H}), 6.28(\mathrm{~d}, J=2.1 \mathrm{~Hz}, 1 \mathrm{H})$, $6.08(\mathrm{~d}, J=2.1 \mathrm{~Hz}, 1 \mathrm{H}) ;{ }^{13} \mathrm{C} \mathrm{NMR}\left(75 \mathrm{MHz}, \mathrm{CDCl}_{3}\right) \delta 188.9,138.3,134.5,132.0,131.2,128.5,126.5$. Anal calcd for $\mathrm{C}_{9} \mathrm{H}_{6} \mathrm{BrClO}: \mathrm{C}, 44.03 ; \mathrm{H}, 2.46$. Found: $\mathrm{C}, 43.88 ; \mathrm{H}, 2.48$.

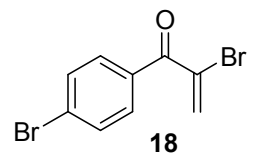

TLC $R_{f}=0.49\left(10 \%\right.$ ethyl acetate/hexanes); IR (film from $\left.\mathrm{CH}_{2} \mathrm{Cl}_{2}\right) 3076,1670,1582,1397 \mathrm{~cm}^{-1} ;{ }^{1} \mathrm{H}$ NMR $\left(300 \mathrm{MHz}, \mathrm{CDCl}_{3}\right) \delta 7.68(\mathrm{~d}, J=10.5 \mathrm{~Hz}, 2 \mathrm{H}), 7.62(\mathrm{~d}, J=10.5 \mathrm{~Hz}, 2 \mathrm{H}), 6.52(\mathrm{~d}, J=2.4 \mathrm{~Hz}$, 
$1 \mathrm{H}), 6.44(\mathrm{~d}, J=2.4 \mathrm{~Hz}, 1 \mathrm{H}) ;{ }^{13} \mathrm{C} \mathrm{NMR}\left(75 \mathrm{MHz}, \mathrm{CDCl}_{3}\right) \delta 189.4,134.0,132.1,131.4,130.3,129.2$, 128.7. Anal calcd for $\mathrm{C}_{9} \mathrm{H}_{6} \mathrm{Br}_{2} \mathrm{O}: \mathrm{C}, 37.28 ; \mathrm{H}, 2.09$. Found: $\mathrm{C}, 37.46 ; \mathrm{H}, 1.74$.

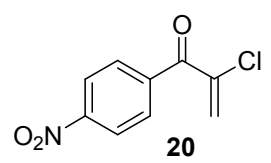

TLC $R_{f}=0.68$ (30\% ethyl acetate/hexanes); $\mathrm{mp}=71-72^{\circ} \mathrm{C}$ (hexanes); IR (film from $\mathrm{CH}_{2} \mathrm{Cl}_{2}$ ) 3110 , 1681, 1602, $1525 \mathrm{~cm}^{-1} ;{ }^{1} \mathrm{H}$ NMR $\left(300 \mathrm{MHz}, \mathrm{CDCl}_{3}\right) \delta 8.33(\mathrm{~d}, J=9.0 \mathrm{~Hz}, 2 \mathrm{H}), 7.94(\mathrm{~d}, J=9.0 \mathrm{~Hz}$, $2 \mathrm{H}), 6.39(\mathrm{~d}, J=2.4 \mathrm{~Hz}, 1 \mathrm{H}), 6.16(\mathrm{~d}, J=2.4 \mathrm{~Hz}, 1 \mathrm{H}) ;{ }^{13} \mathrm{C} \mathrm{NMR}\left(75 \mathrm{MHz}, \mathrm{CDCl}_{3}\right) \delta 188.4,150.5$, 141.1, 138.4, 130.6, 128.1, 123.9. Anal calcd for $\mathrm{C}_{9} \mathrm{H}_{6} \mathrm{ClNO}_{3}: \mathrm{C}, 51.08 ; \mathrm{H}, 2.86 ; \mathrm{N}, 6.62$. Found: $\mathrm{C}$, $51.28 ; \mathrm{H}, 2.85 ; \mathrm{N}, 6.58$.

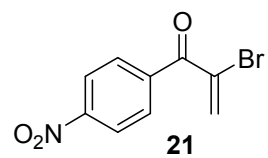

TLC $R_{f}=0.68$ (30\% ethyl acetate/hexanes); $\mathrm{mp}=74-75^{\circ} \mathrm{C}$ (pet. ether);IR (film from $\left.\mathrm{CH}_{2} \mathrm{Cl}_{2}\right) 3117$, 1669, 1591, $1346 \mathrm{~cm}^{-1} ;{ }^{1} \mathrm{H}$ NMR $\left(300 \mathrm{MHz}, \mathrm{CDCl}_{3}\right) \delta 8.33(\mathrm{~d}, J=8.4 \mathrm{~Hz}, 2 \mathrm{H}), 7.95(\mathrm{~d}, J=8.4 \mathrm{~Hz}$, 2H), $6.68(\mathrm{~d}, J=2.7 \mathrm{~Hz}, 1 \mathrm{H}), 6.56(\mathrm{~d}, J=2.7 \mathrm{~Hz}, 1 \mathrm{H}) ;{ }^{13} \mathrm{C} \mathrm{NMR}\left(75 \mathrm{MHz}, \mathrm{CDCl}_{3}\right) \delta 188.6,150.3$, 140.5, 132.3, 130.6, 129.1, 123.8. Anal calcd for $\mathrm{C}_{9} \mathrm{H}_{6} \mathrm{BrNO}_{3}: \mathrm{C}, 42.22 ; \mathrm{H}, 2.36$; N, 5.47. Found: $\mathrm{C}$, $42.60 ; \mathrm{H}, 2.72 ; \mathrm{N}, 5.56$.

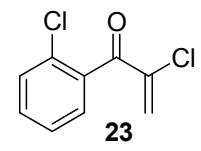

TLC $R_{f}=0.31\left(10 \%\right.$ ethyl acetate/hexanes); IR (film from $\left.\mathrm{CH}_{2} \mathrm{Cl}_{2}\right) 2923,1734 \mathrm{~cm}^{-1} ;{ }^{1} \mathrm{H}$ NMR (300 $\left.\mathrm{MHz}, \mathrm{CDCl}_{3}\right) \delta$ 7.41-7.49 (m, 2H), 7.32-7.40 (m, 2H), $6.39(\mathrm{~d}, J=2.4 \mathrm{~Hz}, 1 \mathrm{H}), 6.07(\mathrm{~d}, J=2.4 \mathrm{~Hz}$, 
$1 \mathrm{H}) ;{ }^{13} \mathrm{C} \mathrm{NMR}\left(75 \mathrm{MHz}, \mathrm{CDCl}_{3}\right) \delta 189.0,140.0,137.3,132.3,131.9,130.4,129.7,129.0,126.9$. Anal calcd for $\mathrm{C}_{9} \mathrm{H}_{6} \mathrm{Cl}_{2} \mathrm{O}: \mathrm{C}, 53.77 ; \mathrm{H}, 3.01$. Found: $\mathrm{C}, 53.39 ; \mathrm{H}, 3.21$.

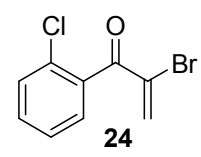

TLC $R_{f}=0.50$ (20\% ethyl acetate/hexanes); IR (film from $\mathrm{CH}_{2} \mathrm{Cl}_{2}$ ) 3108, 3066, $1687 \mathrm{~cm}^{-1} ;{ }^{1} \mathrm{H}$ NMR $\left(300 \mathrm{MHz}, \mathrm{CDCl}_{3}\right) \delta$ 7.42-7.44 (m, 2H), 7.33-7.35 (m, 2H), $6.68(\mathrm{~d}, J=2.7 \mathrm{~Hz}, 1 \mathrm{H}), 6.47(\mathrm{~d}, J=2.7$ $\mathrm{Hz}, 1 \mathrm{H}) ;{ }^{13} \mathrm{C} \mathrm{NMR}\left(75 \mathrm{MHz}, \mathrm{CDCl}_{3}\right) \delta 189.4,136.8,134.7,132.1,132.0,131.6,130.6,129.3,127.1$. Anal calcd for $\mathrm{C}_{9} \mathrm{H}_{6} \mathrm{BrClO}$ : C, 44.03; H, 2.46. Found: C, 44.19; H, 2.49 .

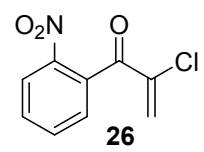

TLC $R_{f}=0.30$ (30\% ethyl acetate/hexanes); IR (film from $\mathrm{CH}_{2} \mathrm{Cl}_{2}$ ) 3370, 3109, 3033, 2924, 2864, 1696, $1606 \mathrm{~cm}^{-1} ;{ }^{1} \mathrm{H}$ NMR $\left(300 \mathrm{MHz}, \mathrm{CDCl}_{3}\right) \delta 8.25(\mathrm{dd}, J=7.5,1.5 \mathrm{~Hz}, 1 \mathrm{H}), 7.76-7.82(\mathrm{~m}, 1 \mathrm{H})$, 7.67-7.72 (m, 1H), $7.58(\mathrm{dd}, J=7.5,1.5 \mathrm{~Hz}, 1 \mathrm{H}), 6.19(\mathrm{~d}, J=2.1 \mathrm{~Hz}, 1 \mathrm{H}), 6.03(\mathrm{~d}, J=2.1 \mathrm{~Hz}, 1 \mathrm{H}) ;{ }^{13} \mathrm{C}$ $\operatorname{NMR}\left(75 \mathrm{MHz}, \mathrm{CDCl}_{3}\right) \delta 187.4,145.5,139.4,134.7,134.6,131.4,128.9,126.7,124.7$. Anal calcd for $\mathrm{C}_{9} \mathrm{H}_{6} \mathrm{ClNO}_{3}: \mathrm{C}, 51.08 ; \mathrm{H}, 2.86 ; \mathrm{N}, 6.62$. Found: $\mathrm{C}, 51.22 ; \mathrm{H}, 2.79 ; \mathrm{N}, 6.73$.

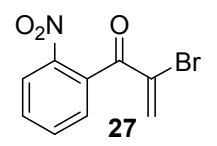

TLC $R_{f}=0.19$ (20\% ethyl acetate/hexanes); $\mathrm{mp}=69-71^{\circ} \mathrm{C}\left(\mathrm{CH}_{2} \mathrm{Cl}_{2}\right)$; IR (film from $\left.\mathrm{CH}_{2} \mathrm{Cl}_{2}\right) 3108$, $1692 \mathrm{~cm}^{-1} ;{ }^{1} \mathrm{H}$ NMR $\left(300 \mathrm{MHz}, \mathrm{CDCl}_{3}\right) \delta 8.23(\mathrm{~d}, J=8.1 \mathrm{~Hz}, 1 \mathrm{H}), 7.80(\mathrm{t}, J=7.2 \mathrm{~Hz}, 1 \mathrm{H}), 7.71(\mathrm{t}, J=$ $7.2 \mathrm{~Hz}, 1 \mathrm{H}), 7.47(\mathrm{~d}, J=7.2 \mathrm{~Hz}, 1 \mathrm{H}), 6.52(\mathrm{~d}, J=2.4 \mathrm{~Hz}, 1 \mathrm{H}), 6.37(\mathrm{~d}, J=2.4 \mathrm{~Hz}, 1 \mathrm{H}) ;{ }^{13} \mathrm{C}$ NMR $(75$ 
$\left.\mathrm{MHz}, \mathrm{CDCl}_{3}\right) \delta 187.5,146.5,134.5,134.3,131.8,131.4,131.2,128.9,124.8$. Anal calcd for $\mathrm{C}_{9} \mathrm{H}_{6} \mathrm{BrNO}_{3}: \mathrm{C}, 42.22 ; \mathrm{H}, 2.36, \mathrm{~N}$, 5.47. Found: $\mathrm{C}, 42.39 ; \mathrm{H}, 2.04, \mathrm{~N}, 5.51$.

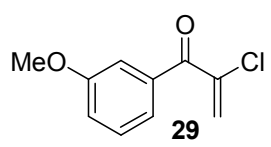

TLC $R_{f}=0.50\left(60 \%\right.$ ethyl acetate/hexanes); IR (film from $\left.\mathrm{CH}_{2} \mathrm{Cl}_{2}\right) 3001,2940,1674,1277 \mathrm{~cm}^{-1} ;{ }^{1} \mathrm{H}$ NMR (300 MHz, $\left.\mathrm{CDCl}_{3}\right) \delta$ 7.36-7.40 (m, 2H), 7.31-7.33 (m, 1H), 7.10-7.19 (m, 1H), $6.29(\mathrm{~d}, J=2.1$ $\mathrm{Hz}, 1 \mathrm{H}), 6.11(\mathrm{~d}, J=2.1 \mathrm{~Hz}, 1 \mathrm{H}), 3.87(\mathrm{~s}, 3 \mathrm{H}) ;{ }^{13} \mathrm{C}$ NMR $\left(75 \mathrm{MHz}, \mathrm{CDCl}_{3}\right) \delta 189.4,159.5,138.3$, 136.7, 129.3, 126.3, 122.0, 119.3, 113.9, 55.3. Anal calcd for $\mathrm{C}_{10} \mathrm{H}_{9} \mathrm{ClO}_{2}$ : C, 61.08; H, 4.61. Found: C, 60.99; H, 4.73.

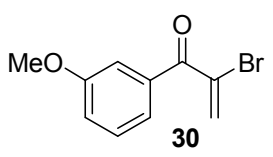

TLC $R_{f}=0.48\left(60 \%\right.$ ethyl acetate/hexanes); IR (film from $\left.\mathrm{CH}_{2} \mathrm{Cl}_{2}\right) 3013,2832,1670,1272 \mathrm{~cm}^{-1} ;{ }^{1} \mathrm{H}$ NMR $\left(300 \mathrm{MHz}, \mathrm{CDCl}_{3}\right) \delta$ 7.31-7.37 (m, 3H), 7.11-7.16 (m, 1H), $6.53(\mathrm{~d}, J=2.4 \mathrm{~Hz}, 1 \mathrm{H}), 6.48(\mathrm{~d}, J=$ $2.4 \mathrm{~Hz}, 1 \mathrm{H}), 3.86(\mathrm{~s}, 3 \mathrm{H}) ;{ }^{13} \mathrm{C} \mathrm{NMR}\left(75 \mathrm{MHz}, \mathrm{CDCl}_{3}\right) \delta 190.4,160.0,136.7,130.7,129.9,129.8$, 122.8, 120.0, 114.5, 55.9. Anal calcd for $\mathrm{C}_{10} \mathrm{H}_{9} \mathrm{BrO}_{2}$ : C, 49.82; H, 3.76. Found: C, 49.71; H, 3.82 . 


\section{REFERENCES}

(1) Pangborn, A. B.; Giardello, M. A.; Grubbs, R. H.; Rosen, R. K.; Timmers, F. J. Organometallics 1996, 15, 1518.

(2) Lyothier, I.; Defieber, C.; Carreira, E. M. Angew. Chem. Int. Ed. 2006, 45, 6204.

(3) White, W. N.; Fife, W. K. J. Am. Chem. Soc. 1961, 83, 3846.

(4) Salih, R. G.; Shandala, M. Y. Collect. Czech. Chem. Commun. 1976, 41, 262.

(5) Lehmann, J.; Lloyd-Jones, G. C. Tetrahedron 1995, 51, 8863.

(6) Bhaskar, G.; Kumar, V. S.; Rao, B. V. Tetrahedron: Asymmetry 2004, 15, 1279.

(7) Makosza, M.; Urbanska, N.; Chesnokov, A. A. Tetrahedron Lett. 2003, 44, 1473.

(8) Morrill, C.; Grubbs, R. H. J. Am. Chem. Soc. 2005, 127, 2842.

(9) Schmidt, B. J. Org. Chem. 2004, 69, 7672.

(10) Evans, P. A.; Leahy, D. K. J. Am. Chem. Soc. 2003, 125, 8974.

(11) Mordini, A.; Peruzzi, D.; Russo, F.; Valacchi, M.; Reginato, G.; Brandi, A. Tetrahedron 2005, 61,3349 .

(12) Stevens, B. D.; Bungard, C. J.; Nelson, S. G. J. Org. Chem. 2006, 71, 6397.

(13) Lee, P. H.; Lee, S. W.; Seomoon, D. Org. Lett. 2003, 5, 4963.

(14) Katritzky, A. R.; Zhang, G.; Jiang, J. J. Org. Chem. 1995, 60, 7589.

(15) Hon, Y.-S.; Hsu, T.-R.; Chen, C.-Y.; Lin, Y.-H.; Chang, F.-J.; Hsieh, C.-H.; Szu, P.-H. Tetrahedron 2003, 59, 1509.

(16) Wu, Z.; Minhas, G. S.; Wen, D.; Jiang, H.; Chen, K.; Zimniak, P.; Zheng, J. J. Med. Chem. 2004, 47, 3282.

(17) Defacqz, N.; Van, T.-T.; Cordi, A.; Marchand-Brynaert, J. Tetrahedron Lett. 2003, 44, 9111.

(18) Bodwell, G. J.; Li, J.; Miller, D.O. Tetrahedron 1999, 55, 12939.

(19) Hayami, J.; TANaka, N.; Kaji, A. Bull Chem. Soc. Jpn. 1973, 46, 954.

(20) Modaral, B.; Khoshdel, E. J. Org. Chem. 1977, 42, 3527. 

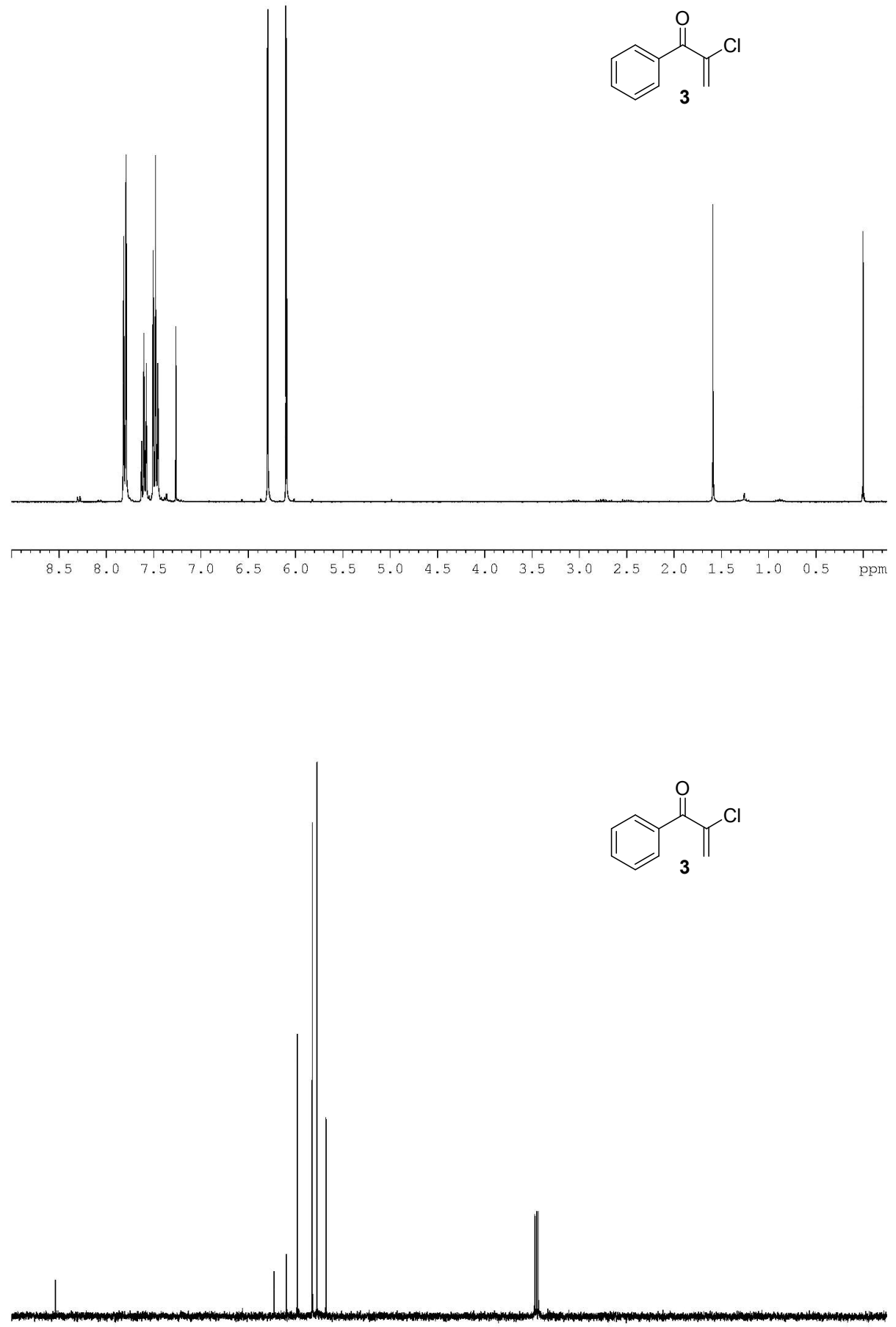

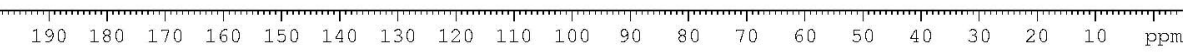



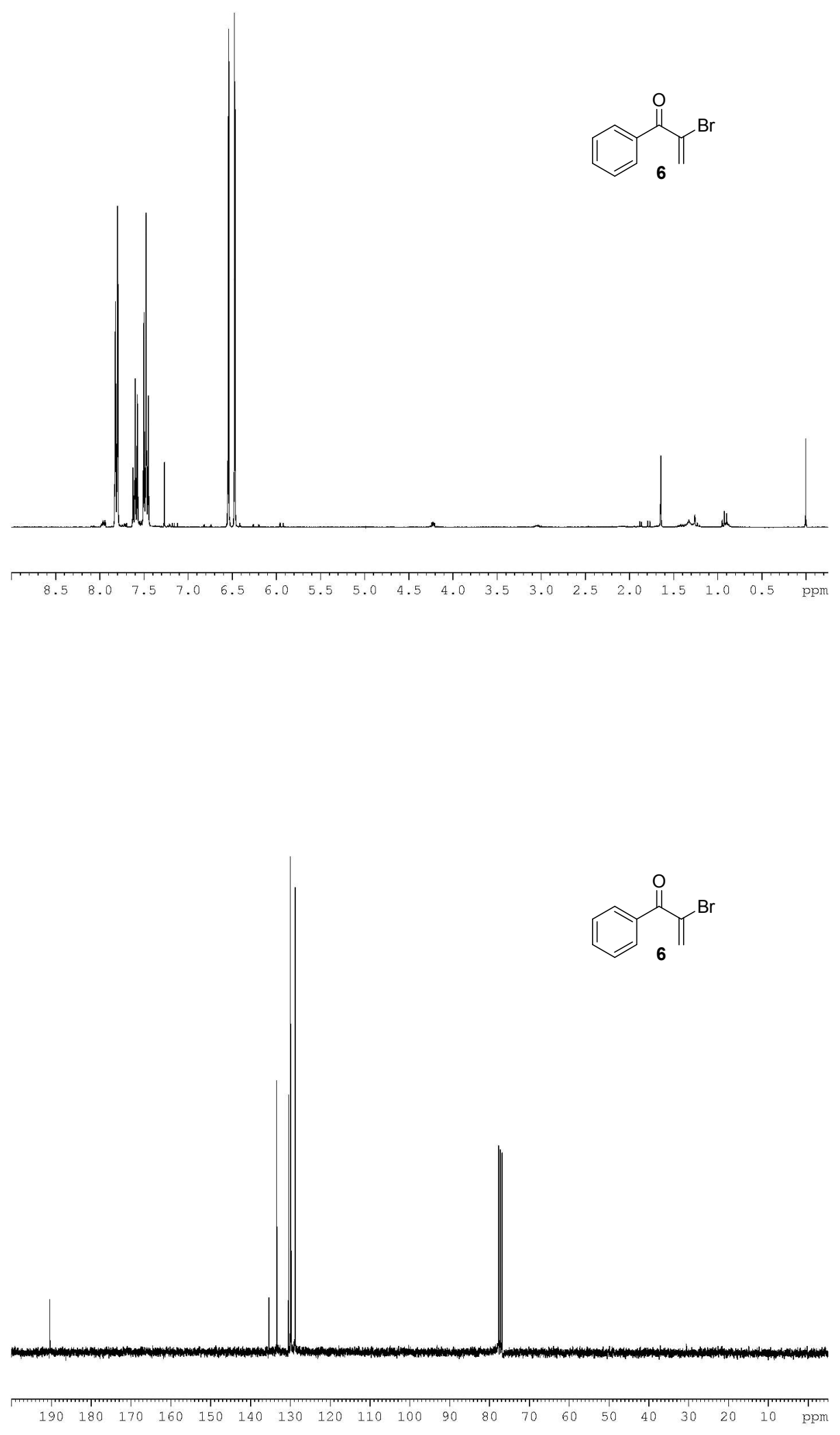

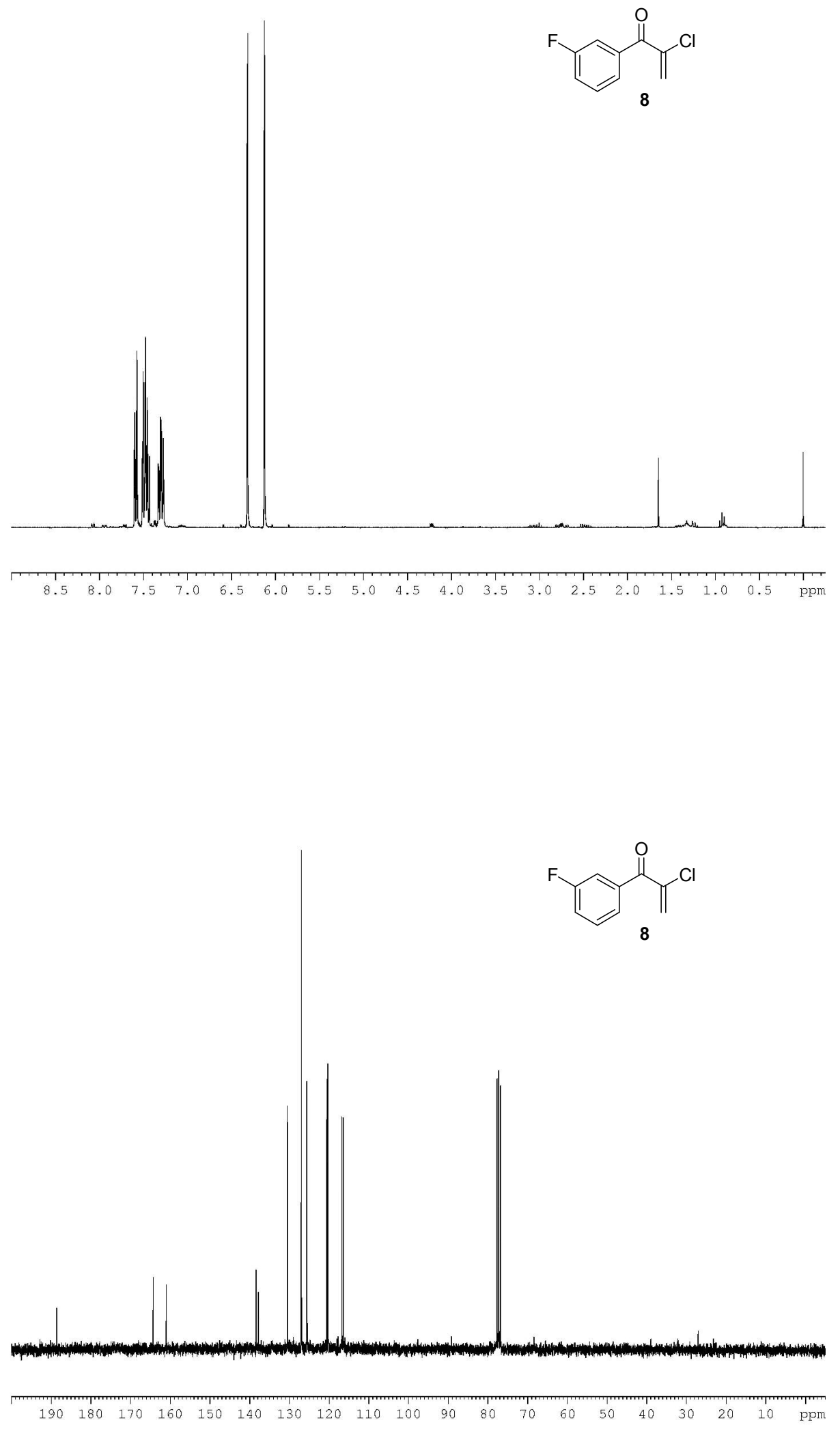

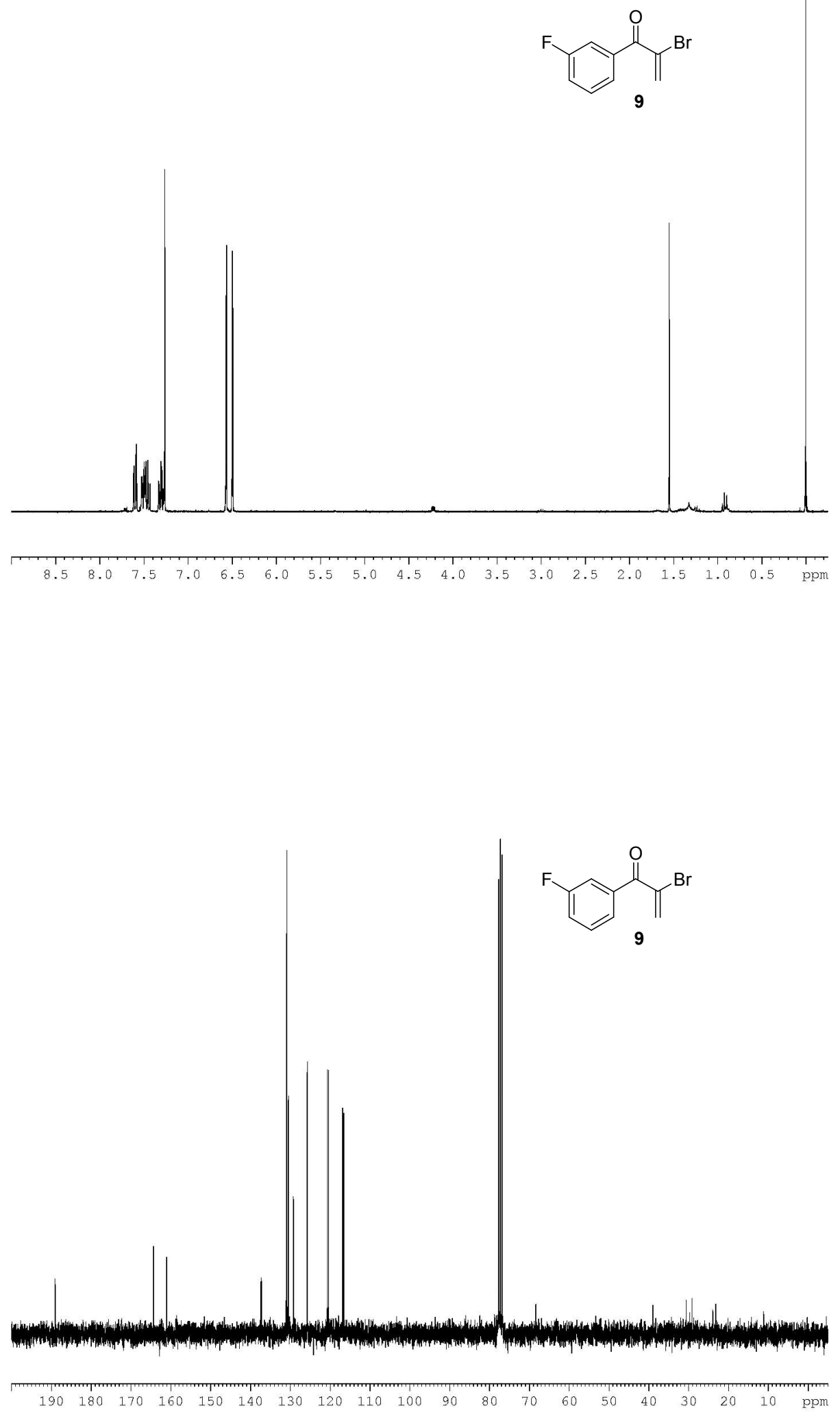

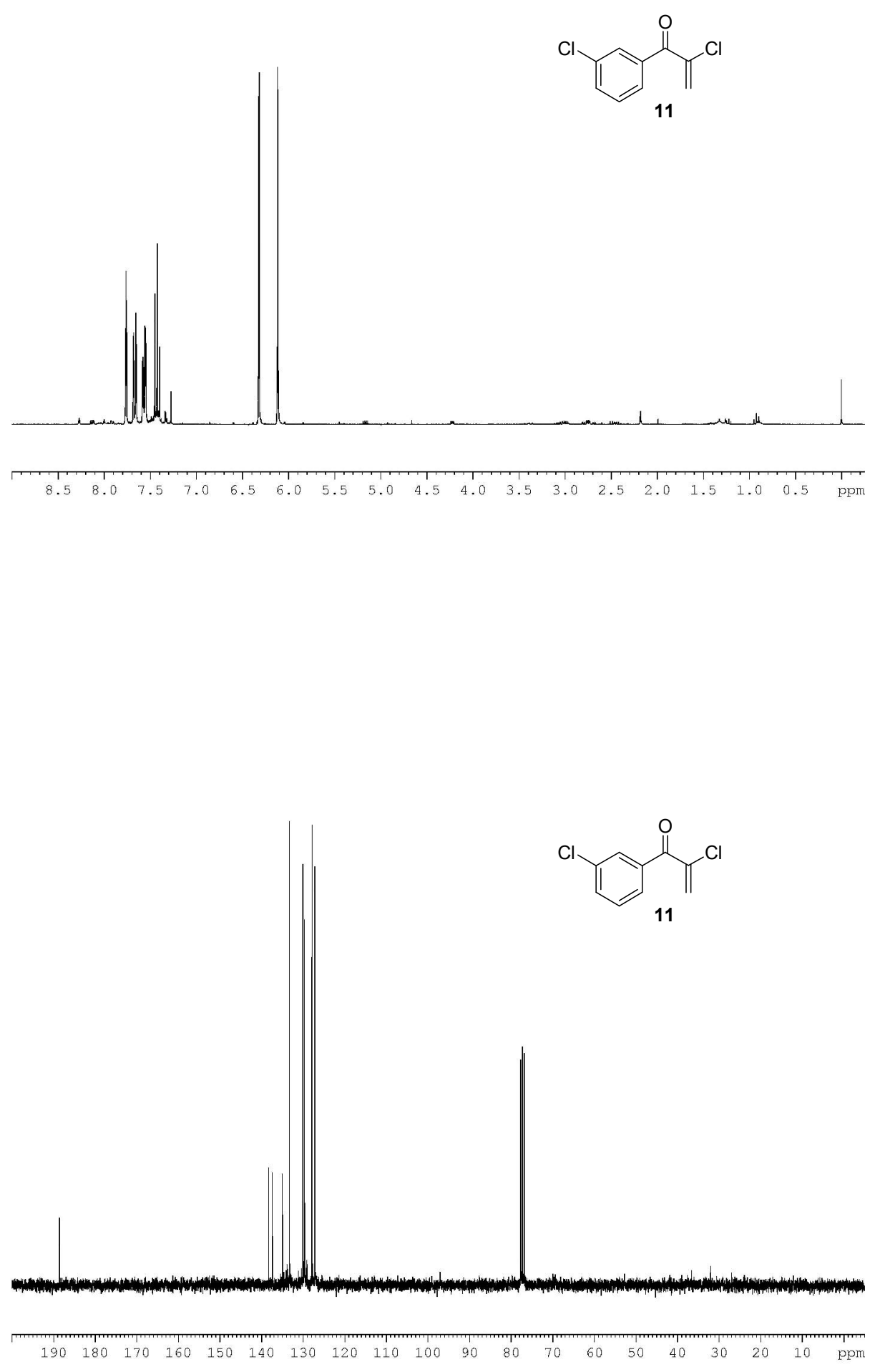

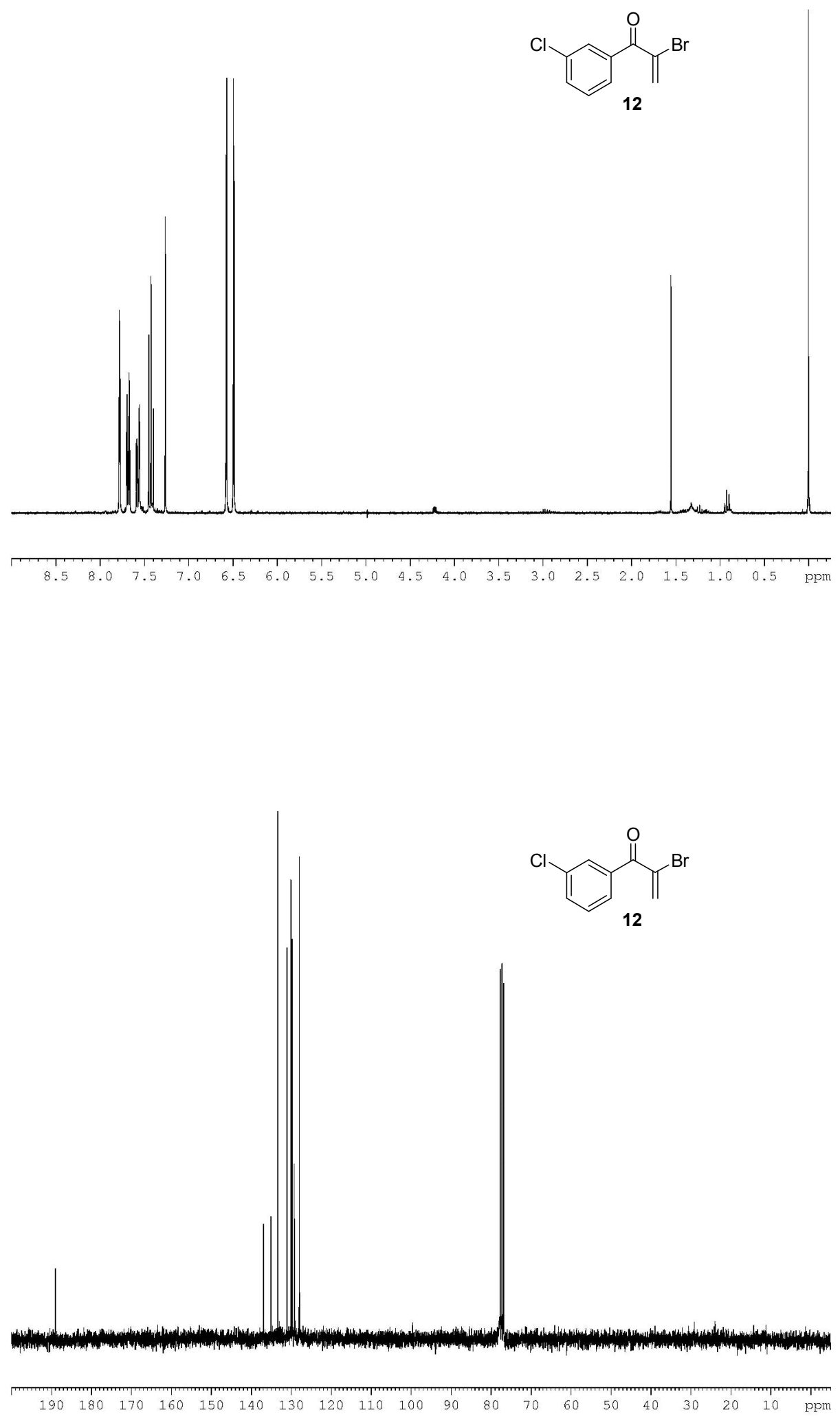

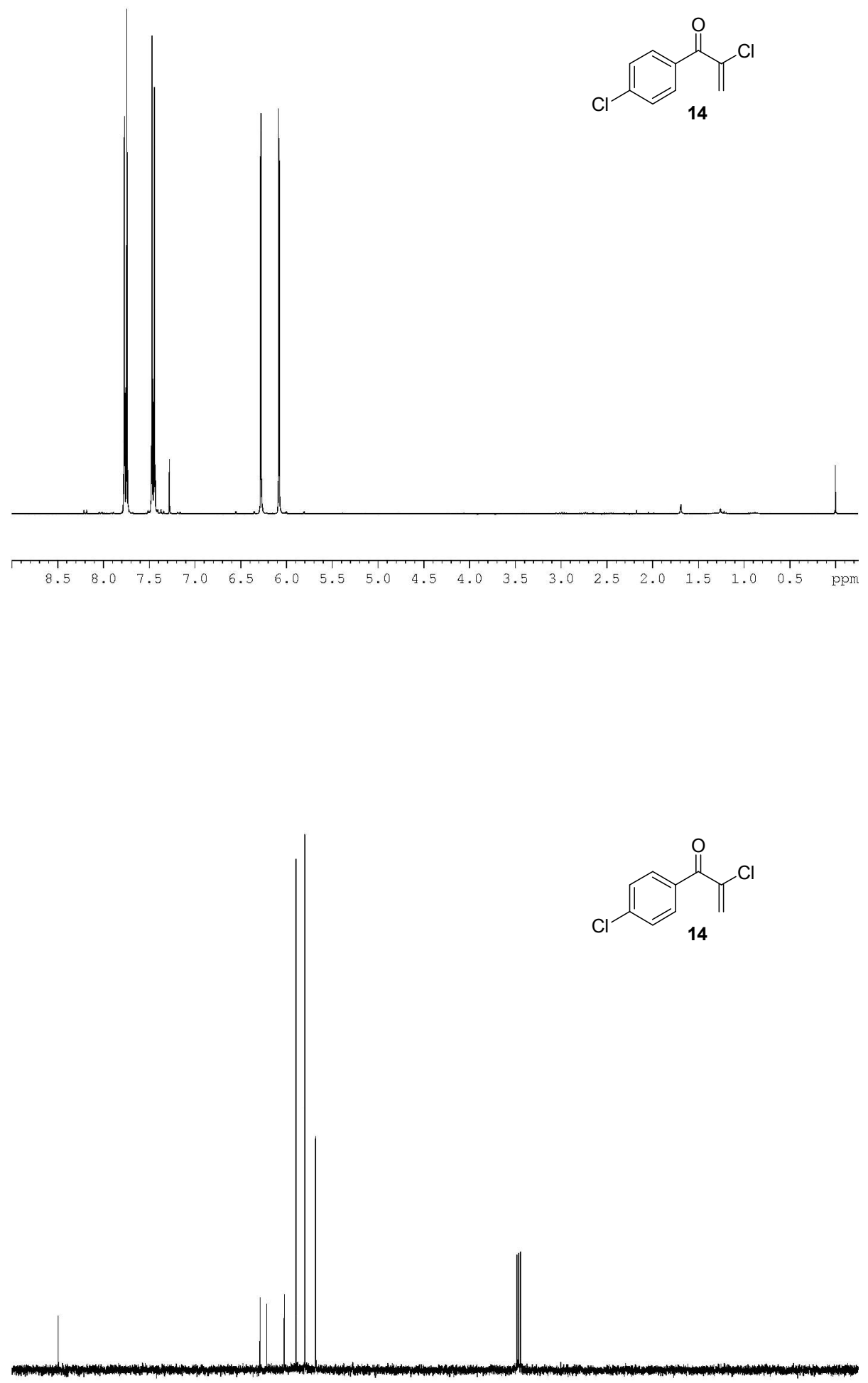

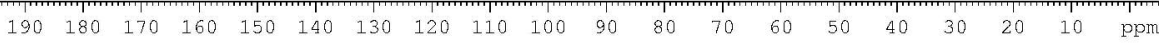



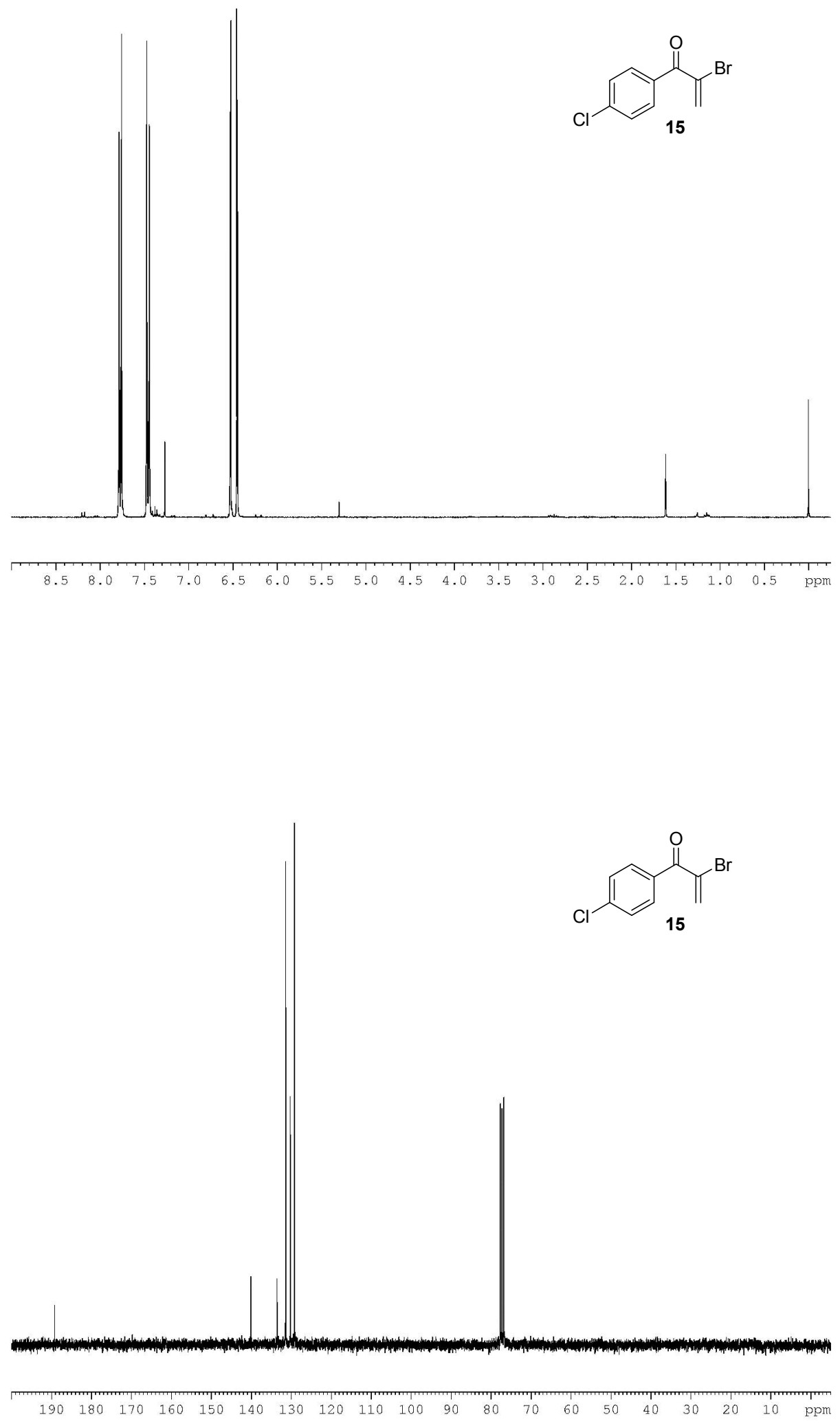

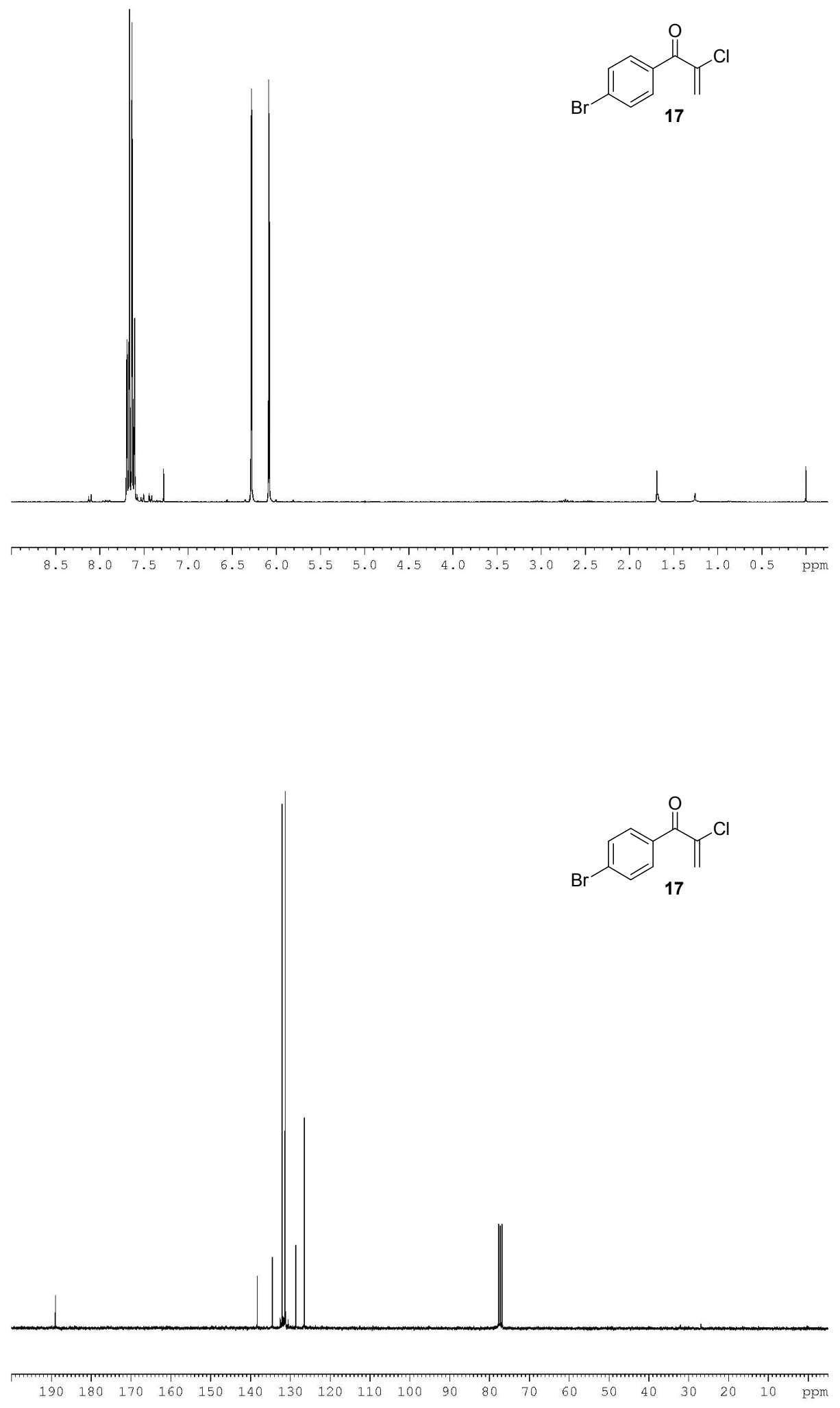

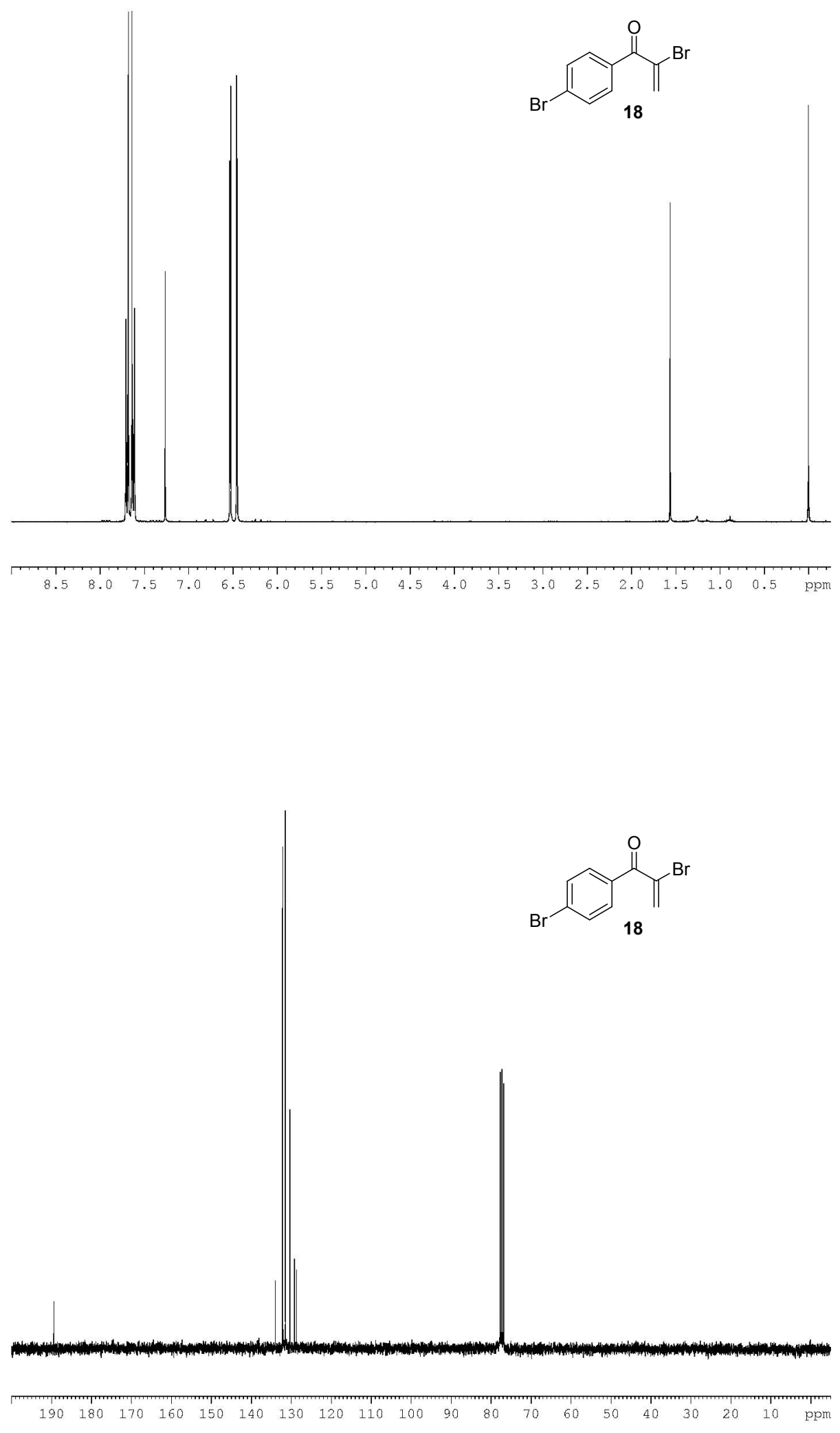

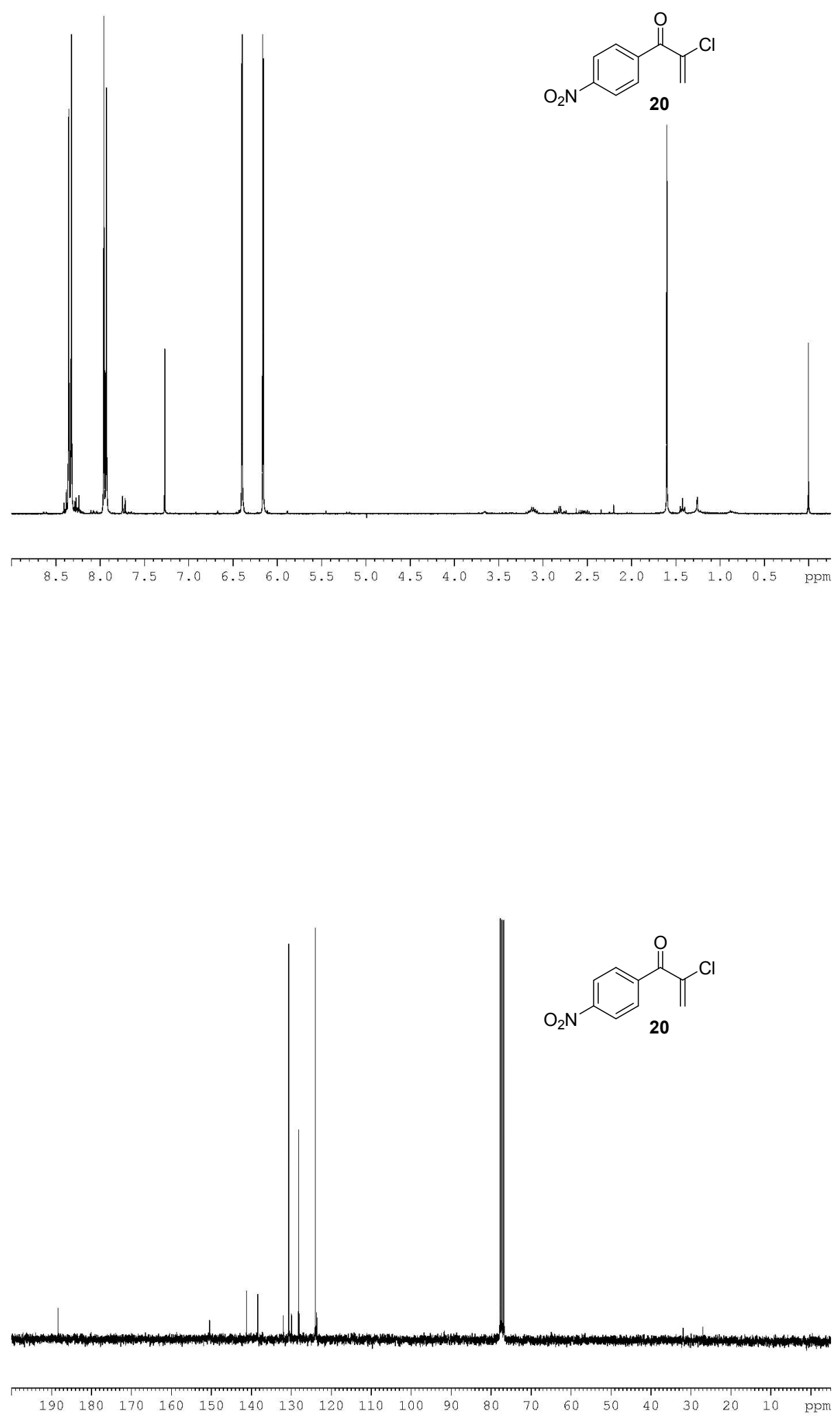

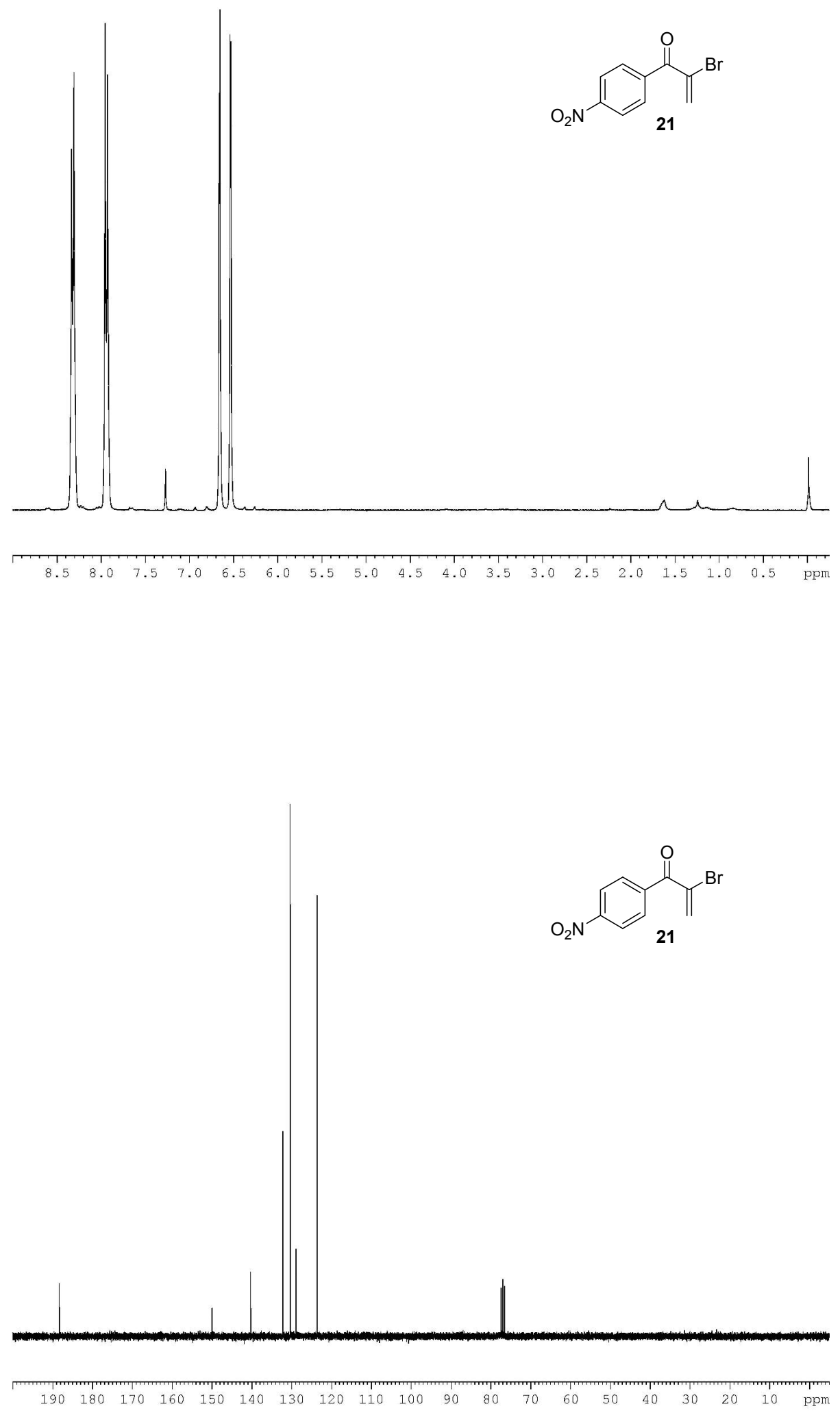


$$
\frac{\|^{*}}{H^{*}}
$$



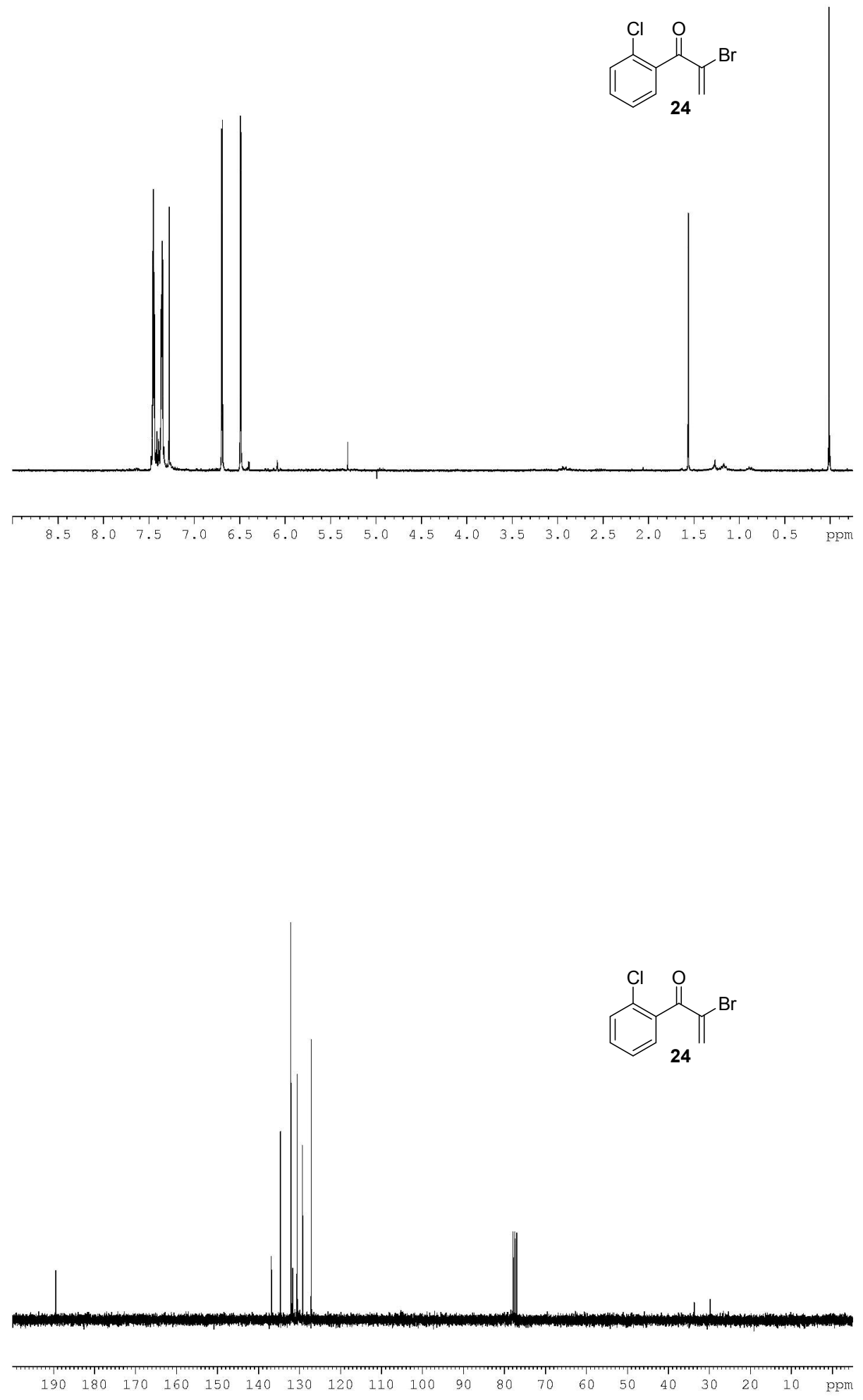

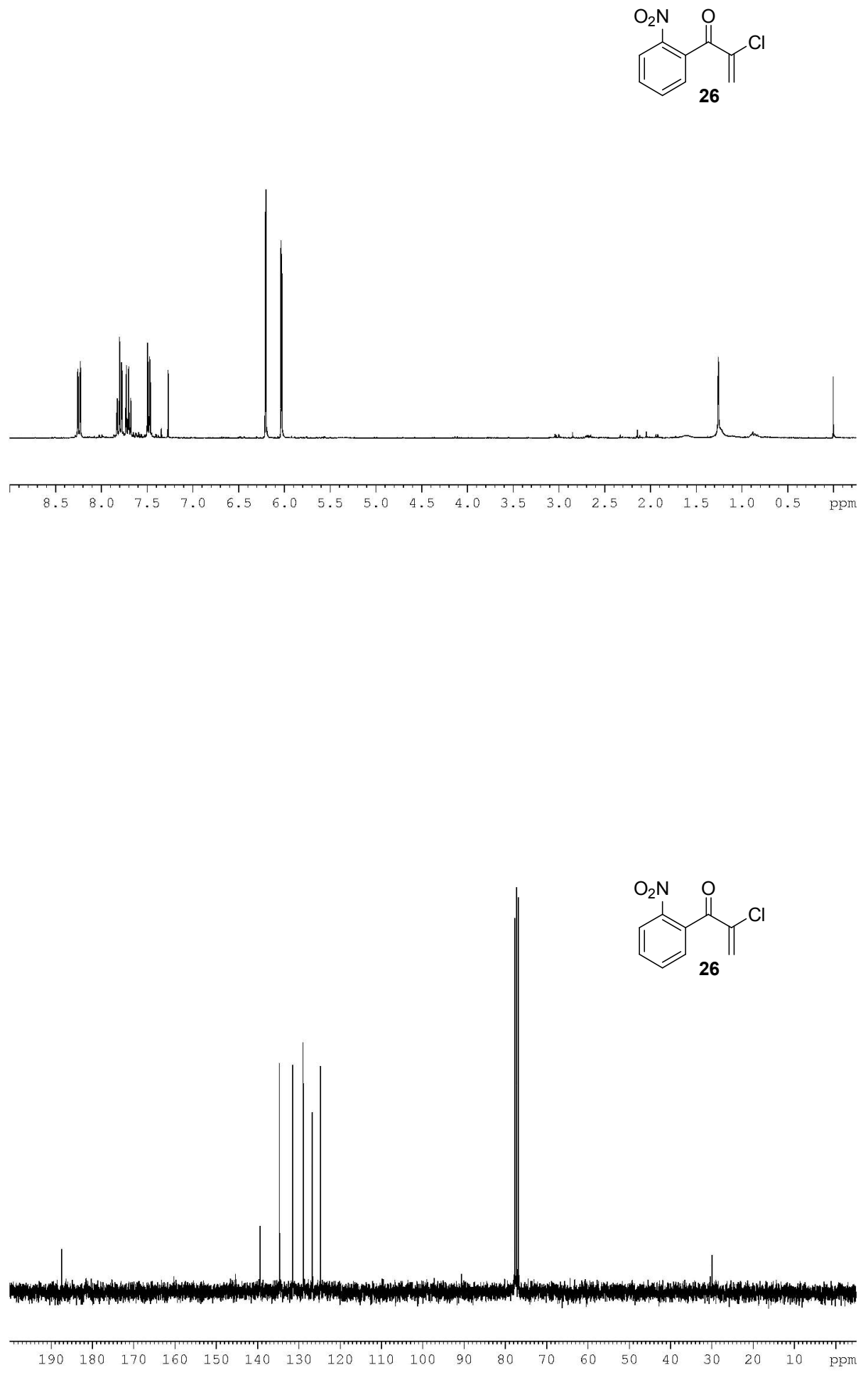

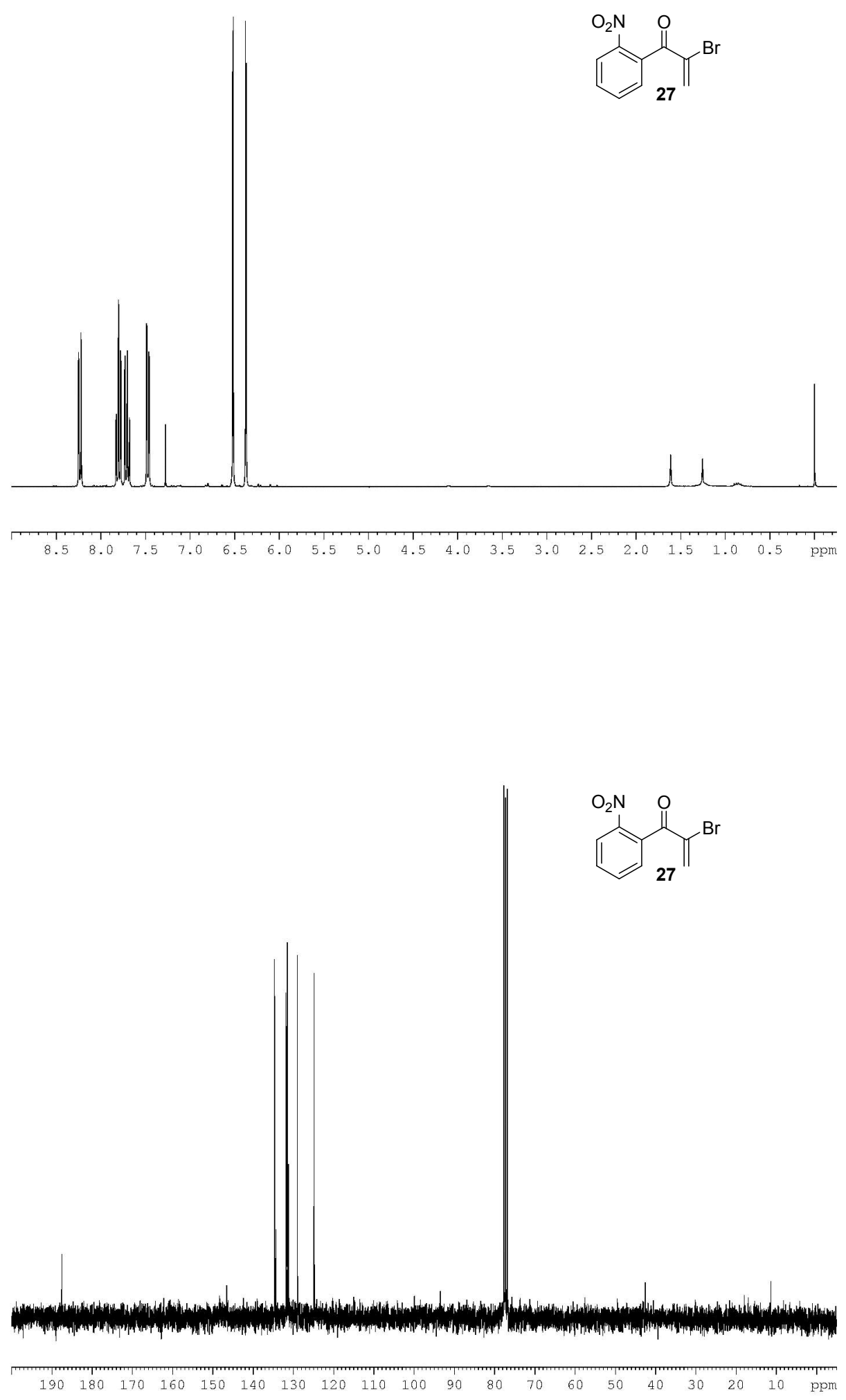

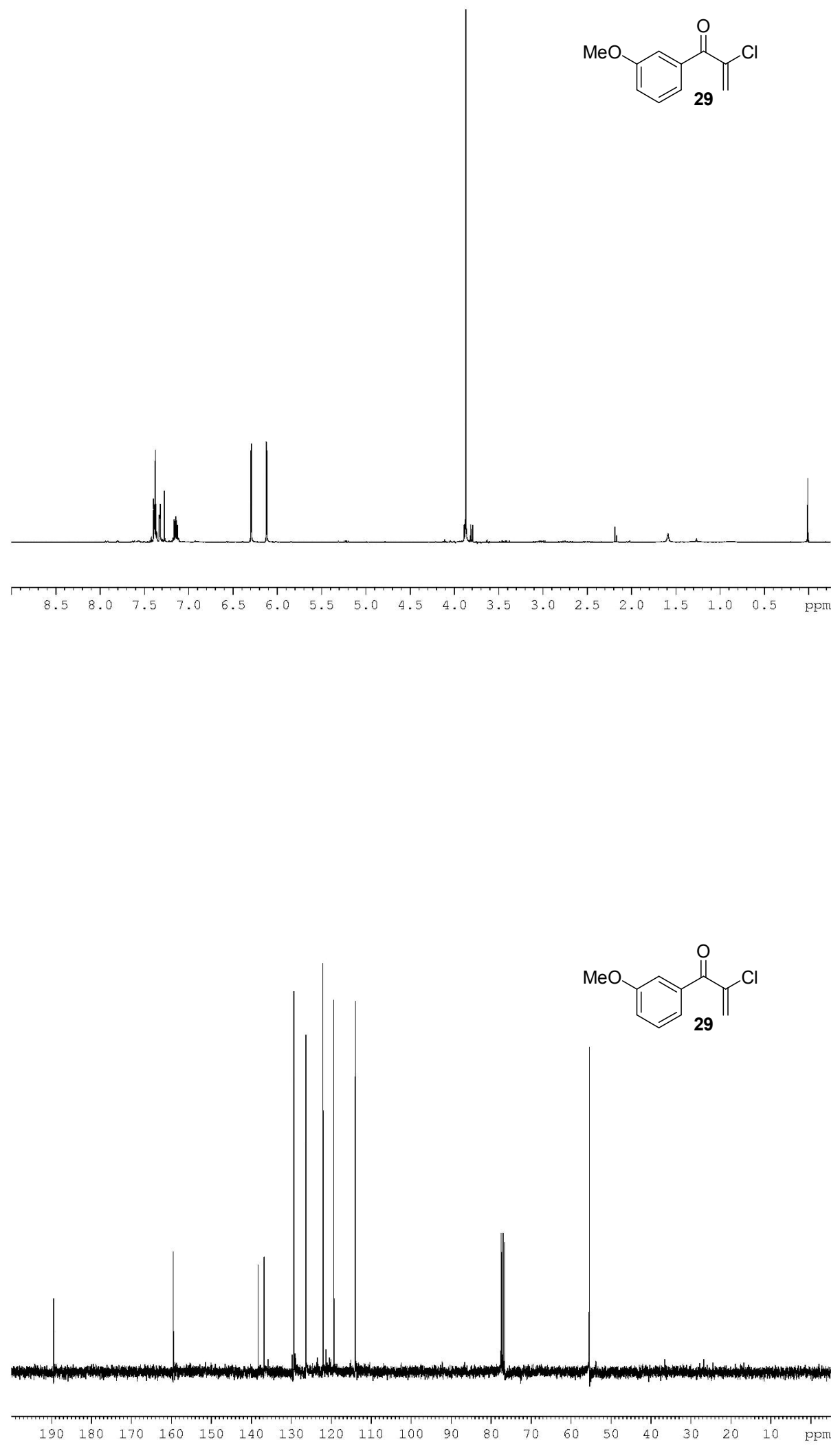

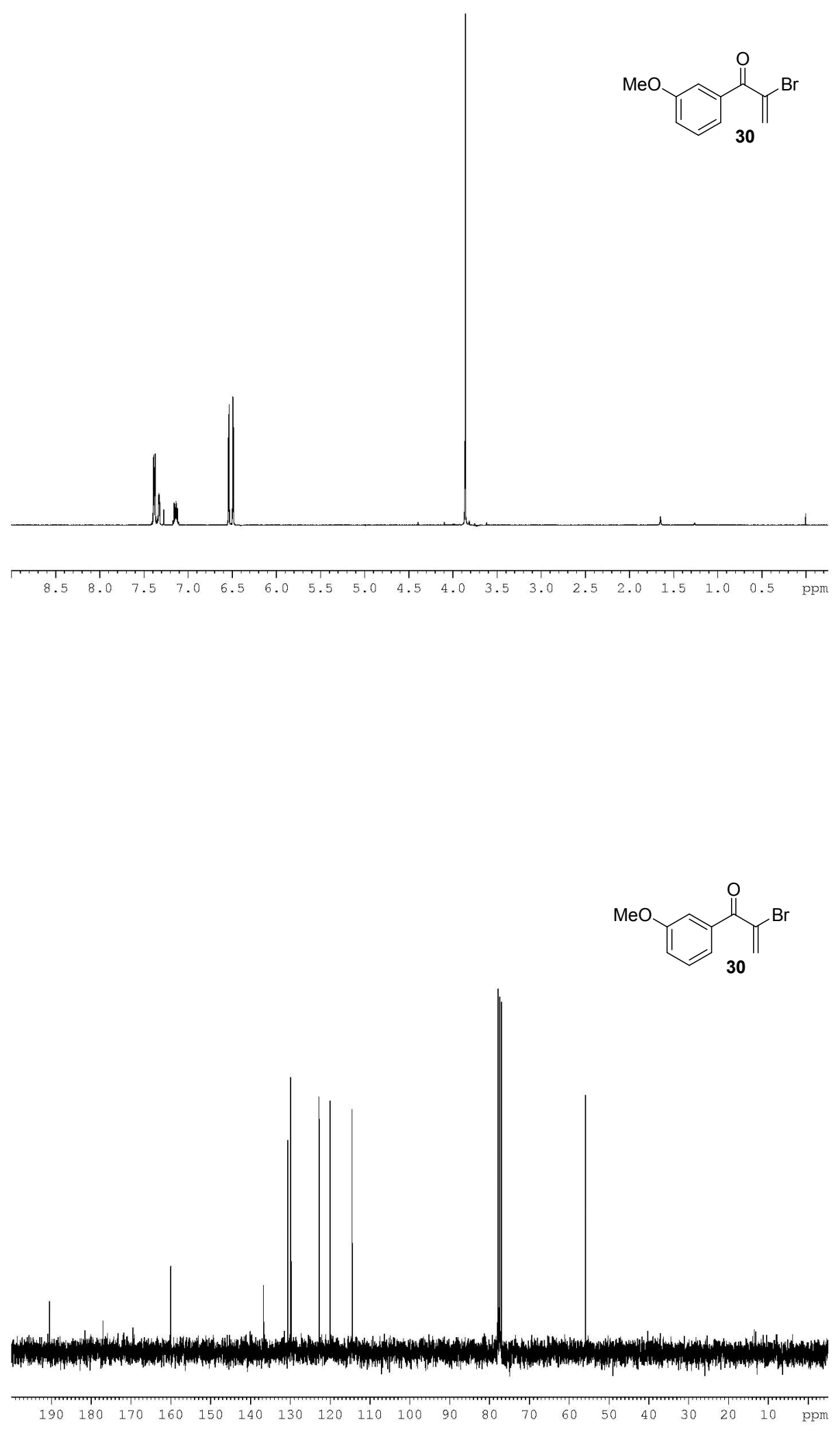

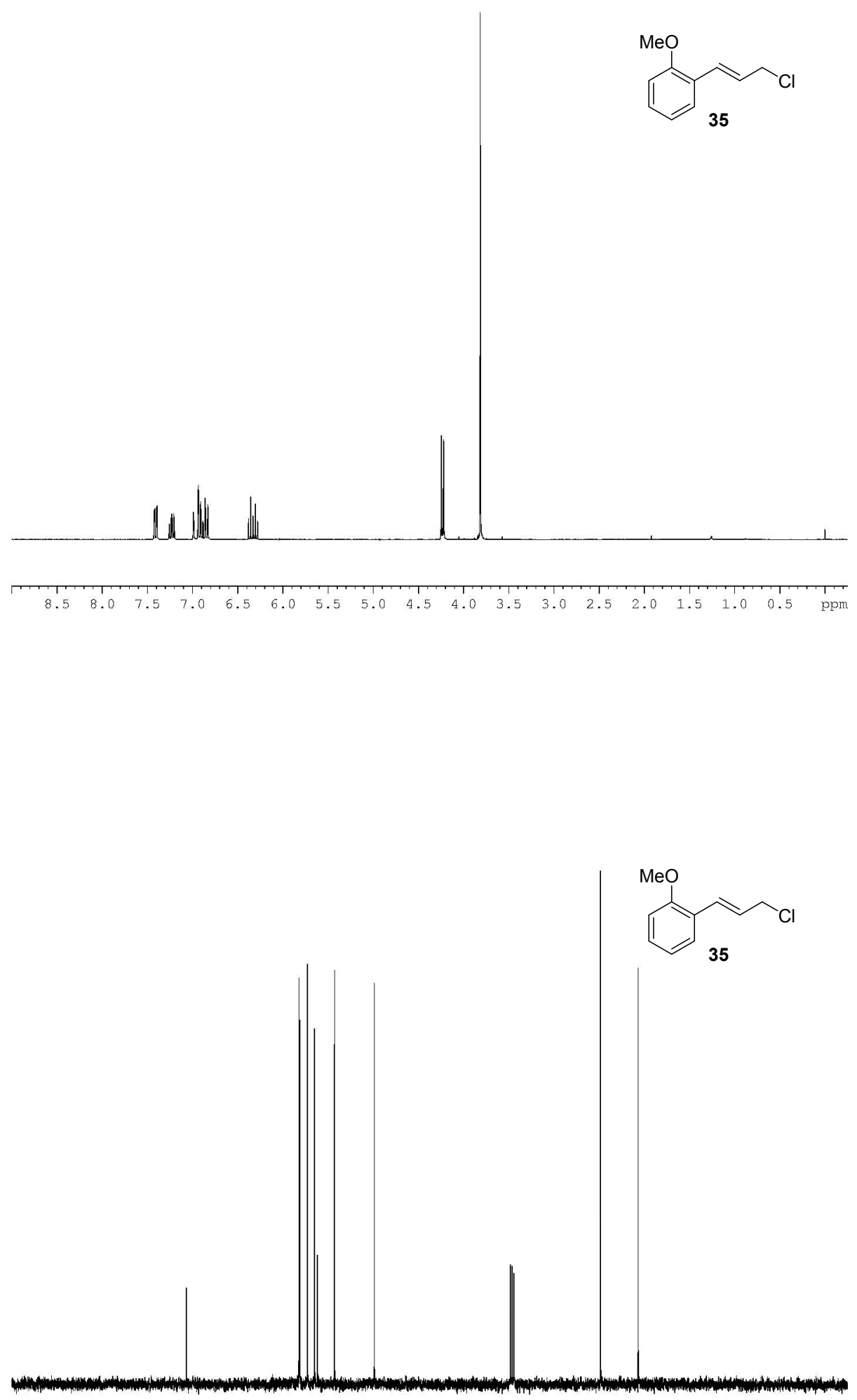